\title{
Dynamics of actin polymerisation during the mammalian single-cell wound healing response
}

\author{
Corina DeKraker, Laurence Goldin-Blais, Eric Boucher and Craig A. Mandato* (1)
}

\begin{abstract}
Objective: The contribution of actomyosin contractile rings in the wound healing program of somatic cells as never been directly assessed. This contrast with the events characterising the wound healing response of in wounded Xenopus oocytes, in which formation and contraction of an actomyosin ring provides a platform for cytoskeletal repair and drives the restoration of proper plasma membrane composition at the site of injury. As such, we aimed to characterize, using high-resolution live-cell confocal microscopy, the cytoskeletal repair dynamics of HeLa cells.

Results: We confirm here that the F-actin enrichment that characterizes the late repair program of laser-wounded cells is mostly uniform and is not associated with co-enrichment of myosin-ll or the formation of concentric zones of RhoA and Cdc42 activity.
\end{abstract}

Keywords: Single-cell repair, Plasma membrane repair, Cytoskeletal repair, Actin dynamics, Actomyosin ring, Liveconfocal microscopy

\section{Introduction}

Single-cell repair is highly context-dependent. Small, nanometer-sized plasma membrane (PM) wounds are thermodynamically favored to reseal spontaneously [ 1 , 2]. Conversely, the repair of the very large wounds studied in Xenopus laevis and sea urchin oocytes, and in early drosophila embryos, requires the involvement of rapid exocytic events as well as the formation and "explodosis" of a large membrane patch [3]. In the same models, restoration of normal PM composition and of damaged cytoskeletal structures involves the cooperation between the contraction of an actomyosin ring [4] and actin polymerisation [5]. The repair of clinically and physiologically-relevant wounds experienced by somatic cells such as neurons, myofibers, and pneumonocytes also involves exocytic events are followed by the same core steps of wound stabilization: exocytosis of intracellular

*Correspondence: craig.mandato@mcgill.ca

Department of Anatomy and Cell Biology, Faculty of Medicine, McGill University, 3640 University Street, Strathcona Anatomy and Dentistry Bldg, Montreal, QC H3A OC7, Canada vesicles, followed by spontaneous repair, or the removal of damaged PM components by shedding or endocytosis (reviewed in [6]). It is also worth noting that none of these processes are able to fully restore a cell's PM to its pre-wounded state [7], or restore damaged underlying cortex cytoskeletal structures $[8,9]$. Somatic cells possess the necessary machinery to form contractile ring (CR) structures [10], but formation and contraction of a CR following PM injury have never been directly observed or confirmed. In Xenopus oocytes, CRs have been observed in response to puncture wounds of $150 \mu \mathrm{m}$ in diameter [11] and laser wounds of approximately $50-100 \mu \mathrm{m}$ in diameter $[5,12]$. The wounds created in previous assays performed in mammalian cells are usually much smaller, historically in the range of a few $\mu \mathrm{m}$ in diameter. Is the smaller nature of the wounds created in somatic cells the reason for the apparent absence of a CR upon wounding?

We present here the results of a wound healing assay aiming to answer these questions and to directly investigate the presence of an CR following PM injury in somatic cells. Data was acquired by way of a laser-mediated wounding assay which created very large $(10 \mu \mathrm{m}$ 
range) wounds on the bottom surface of HeLa cells. We then imaged the PM and cytoskeletal repair dynamics using high-resolution, live confocal microscopy. We report strong microscopic evidence confirming that the wound healing response program of mammalian cells is mostly driven via actin polymerisation and does not necessarily involve the presence of a CR.

\section{Main text \\ Materials and methods Cell culture}

HeLa cells purchased from ATCC (ATCC CCL-2) were maintained at $37{ }^{\circ} \mathrm{C}$ with $5 \% \mathrm{CO}_{2}$, in MEM, supplemented with $10 \%$ FBS, $1 \%$ Penicillin-Streptomycin, and 1\% Glutamax (all reagents from Gibco, Canada). For live imaging, cells were seeded on glass-bottom dishes (1.5 thickness; MatTek, USA) and imaged in phenol red-free imaging media.

\section{PM labelling and targeted laser ablation of the PM}

CellMask Deep Red Plasma Membrane Stain (Invitrogen, USA) was used to label the PM as per the manufacturer's instructions and was used to target multiple sequential laser pulses of a MicroPoint UV laser (Andor, Northern Ireland) to a $\sim 95 \mu^{2}(11 \mu \mathrm{m}$ diameter) area of the PM on the side contacting the dish (i.e. basal side). The success of PM ablation was made on the basis of the creation of a visually detectable gap in the fluorescent signal for both the PM (CellMask) and cytoskeletal compartments. Formation of bona fide PM and cytoskeletal wounds (vs photobleaching) was confirmed via a series of independent wounding/repair. FM1-43 (N-(3Triethylammoniumpropyl)-4-(4-(Dibutylamino) Styryl) Pyridinium Dibromide; Invitrogen, USA) wounding assays [13] (Additional file 1), and re-assessed at regular intervals.

\section{Transfection of genetically-encoded probes for live imaging}

Cytoskeletal dynamics and RhoGTPase activity were tracked using a series of genetically encoded probes: F-actin (calponin homology domain of utrophin; PCS2UtrCH-GFP; Addgene \#26737 or PCS2-UtrCH-mCherry; Addgene \#26740), myosin-II (RFP-tagged myosin regulatory light chain 1; PCS2-MRLC-RFP), microtubules (GFP-labelled microtubule binding-domain of ensconsin; PCS2-EMTB-3xGFP; Addgene \# 26741), active RhoA (RhoA-binding domain of rhotekin; PCS2- $\gamma$ GBD-GFP; Addgene \#26732), and Cdc42 (Cdc42 binding domain of N-WASP; PCS2- $\omega$ GBD-GFP Addgene \# 26734). All plasmids were transfected using PolyJet In Vitro DNA transfection reagent (SignaGen, USA), as per the manufacturer's instructions.

\section{Imaging}

Imaging was performed at the Cell Imaging and Analysis Network (CIAN) imaging facility at McGill University, using a Quorum WaveFX-X1 spinning disk confocal system on a Leica DMI6000B inverted microscope, two Hamamatsu "ImagEM X2 C9100-23B" EM-CCD cameras, and an $63 \times / 1.40$ oil objective (lBL, HCX PL APO; 506192). Cells were kept at $37{ }^{\circ} \mathrm{C}$ with $5 \% \mathrm{CO}_{2}$ using a Live Cell Instruments Chamlide TC environmental control system. Acquisition was performed using MetaMorph Microscopy Automation and Image Analysis Software (Molecular Devices LLC, USA) and consisted of Z-stacks (11 slices, $0.2 \mu \mathrm{m}$ apart), taken every $10 \mathrm{~s}$ for at least 15 min. $1 \times 1$ binning, 1 gain, and $100 \mathrm{EM}$ gain was used throughout.

Images were further processed using ImageJ software (National Institutes of Health, USA). Quantitation of fluorescence at the wound site, was performed on maximum intensity projections ( 3 slices, $0.2 \mu \mathrm{m}$ apart). Average fluorescence intensity was measured at every 10 -s time point, at four $95 \mu \mathrm{m}^{2}$ sites: the wound site region $\left(\mathrm{FI}_{\mathrm{W}}\right)$ and three control cytoplasmic regions within the cell $\left(\mathrm{FI}_{\mathrm{C}}\right)$. The relative fluorescence intensity at the wound site $\left(\mathrm{FI}_{\mathrm{R}}\right)$ was calculated by dividing $\mathrm{FI}_{\mathrm{W}}$ by the average of the three $\mathrm{FI}_{\mathrm{C}}$. To enable cell-to-cell comparisons, the resulting $\mathrm{FI}_{\mathrm{R}}$ were then further normalized to the average $\mathrm{FI}_{\mathrm{R}}$ on the same unwounded cell. Micrographs, kymographs, and orthogonal views were prepared from maximum intensity projections (MIPs) of the entire imaged volume (10 slices, $0.2 \mu \mathrm{m}$ apart).

\section{Statistics}

Statistical analyses were performed using GraphPad Prism 7.04 (GraphPad Software Inc., USA) and consisted of repeated measure 2-way ANOVAs, followed by Dunnett's multiple comparisons tests. Significance was set at $\mathrm{p}<0.005$.

\section{Results and discussion}

We provide here substantial evidence that under normal 2D-culture conditions, mammalian cytoskeletal repair or large PM and cytoskeletal injuries involves actin polymerisation, without the formation of actomyosin contractile arrays.

\section{Wound healing response involves uniform actin polymerisation, but not myosin-II accumulation}

We first set out to also track the spatio-temporal dynamics of actin polymerisation that follows laser-mediated injury of HeLa cells. As expected, the ablated area showed clear indications of actin polymerisation following wounding (Figs. 1 and 3a; Additional file 2, Additional 


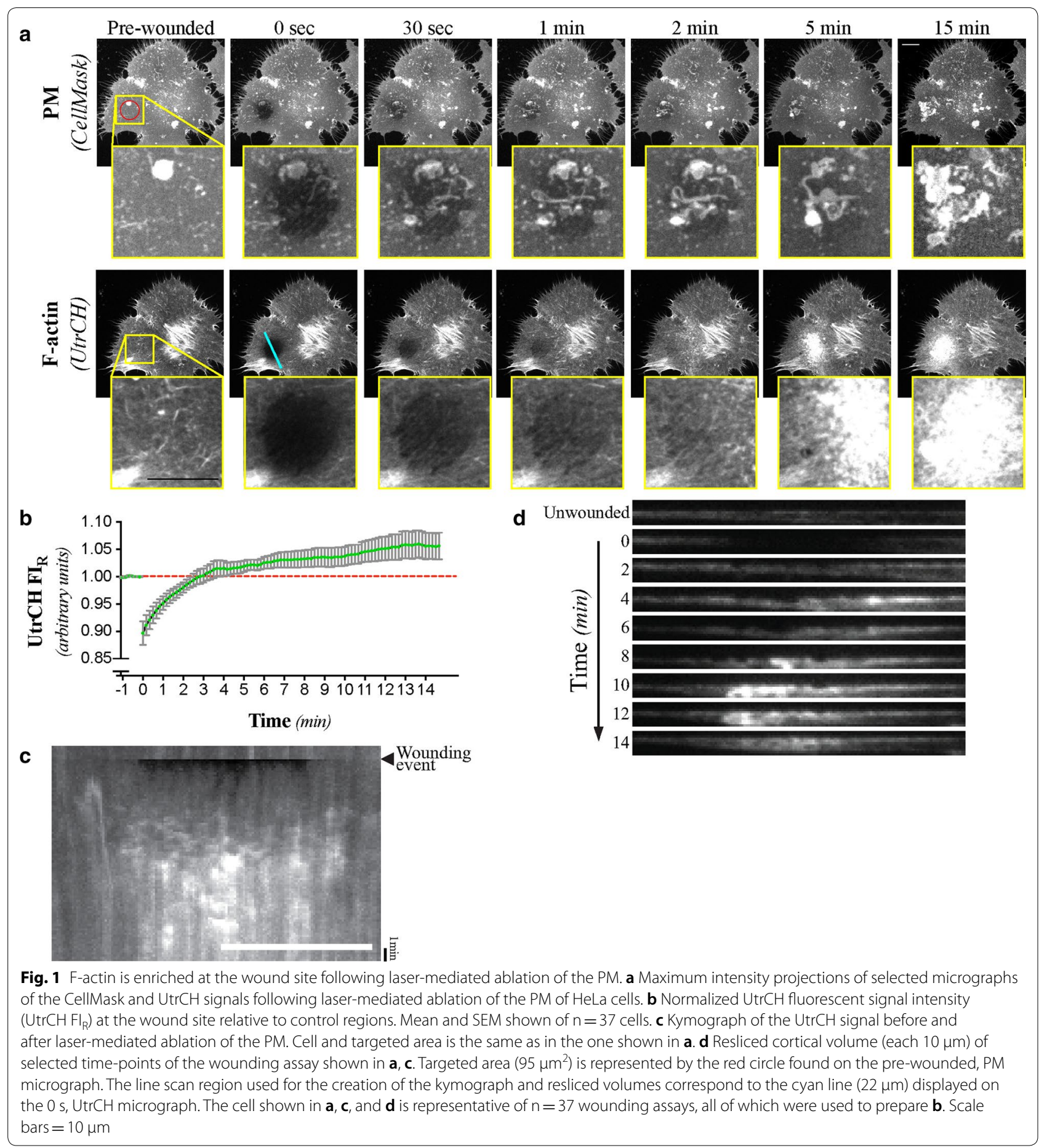

file 3, Additional file 4, Additional file 5, Additional file 6, Additional file 7, Additional file 8 and Additional file 9). Furthermore, UtrCH $\mathrm{FI}_{\mathrm{R}}$ rapidly rose above the baseline post-wounded levels (significant after $30 \mathrm{~s}$ ), which was significantly higher than the pre-wounding baseline levels after 6 min (Fig. 1b), suggesting enrichment of F-actin at the wound site. CR-mediated repair involves the formation of two functionally and structurally distinct zones of actin polymerisation: a dense ring of F-actin and Myo$\sin$-II located at the wound edge, which is surrounded by a wider array of perpendicularly oriented F-actin $[5,11$, $14,15]$. Neither F-actin patterns were readily observable 
(Fig. 1a, 3a) during the course of more than 37 individual wounding assays. Kymographic and line scan analyses (Fig. 1c, 3b, c; Additional file 2) confirmed this qualitative assessment and also failed to reveal any other significant spatially defined enrichment of the UtrCH signal. Instead, $\mathrm{UtrCH}$ signal increased uniformly over the entire area of the wound site, with no noticeable enrichment at the wound edge (Fig. 1c, 3c; Additional file 2). Orthogonal views of the wound site were similarly unable to detect any localized UtrCH signal indicative of a clearly defined actomyosin structure (Fig. 1d).

The spatio-temporal dynamics of myosin-II following injury was also investigated (Fig. 2a), as it is responsible for the actomyosin array's contractility [11]. We found that the return of myosin-II at the wound site did not follow the same pattern as we observed for F-actin, as the rate of MRLC $\mathrm{FI}_{\mathrm{RW}}$ signal accumulation to the wound site (Fig. 2b) was much slower than the one observed for $\mathrm{UtrCH}$, and never went above pre-wounded baseline levels within the time allotted (Fig. 2b). Like F-actin, myosin-II did not show sign of accumulating according to any discernible patterns (Fig. 2a, c and d; Additional file 10). The sequence timing of F-actin and of myosin-II accumulation, broadly confirms the findings of other reports for wounds created on both the apical or basal side of cells, using a variety of wounding methods [16-18]. Specific differences in the actin polymerisation dynamics between our and all of the other previous studies could be attributable to the differences in wound size, wounding methods (laser vs mechanical), location (apical vs basal), cell shape and in local tension between the different wounding assays.

\section{The mammalian wound healing response does not involve spatially regulated $\mathrm{RhoA}$ or Cdc42 signalling, microtubule arrays, or coordination of PM restoration and cytoskeletal repair}

The presence of concentric rings of RhoA and Cdc42 activity around the wound edge is a hallmark event of the formation of actomyosin contractile arrays [19]. As such, we set out to characterize the spatio-temporal distribution of RhoA and Cdc42 signalling in the context of our wound healing assay. Neither $\gamma$ GBD (Fig. 3) or $\omega$ GBD (Additional file 3) signals coalesced into the clearly defined RhoA or Cdc42 "activity zones" around the wound edge that would be necessary to the formation and contraction of CR, as demonstrated in wounded Xenopus oocytes [19, 20]. Instead, RhoA activity exhibited a pattern that closely matched that of UtrCH and accumulated over the entire area of the wound site (Fig. 3a, c). YGBD signal quickly rose after wounding, but never rose above its "pre-wounded" baseline levels (Fig. 3b). We were unable to observe any quantifiable or observable change in $\omega \mathrm{GBD}$ following wounding anywhere in the cell, following the wounding event (Additional file 3; Additional file 7). These findings are consistent with a previous study that examined RhoA activity in mouse myoblasts wounded with a single laser blast to the basal side of their PM, which found local RhoA activation signal at the wound site that also failed to show ring-like patterning [18]. The same study attributed this pattern of RhoA activity to the increased reactive oxygen species (ROS) production caused by the increased mitochondrial uptake of calcium through the mitochondrial calcium uniporter (MCU) [18]. Whether mitochondria mediated ROS production also plays a role in the RhoA activation and actin polymerisation events observed in wounds as large as the ones produced within the context of our study remains to be confirmed.

We also failed to observe the formation of a MT array following injury. Instead, MTs seemed to polymerize from the wound-edge inward (Additional file 6; Additional file 11). This would be consistent with the absence of an actomyosin ring as CR formation is further driven by cortical flow and feedback with microtubules (MTs), which accumulate in a radial array around the wound site and the CR in the Xenopus oocyte model $[5,11,12]$. These observations are also compatible with a study of mouse myoblasts wounded with a single laser point, where MTs were also found to be neither enriched nor necessary for repair [18] and with observations about the dynamics of MTs of mechanically wounded fibroblasts [21].

In echinoderm oocytes, Xenopus oocytes, and early Drosophila embryos, PM and cytoskeletal repair are deeply interconnected (reviewed [6]). The contraction of the actomyosin array not only provides a platform for F-actin polymerisation, but also regenerates normal PM composition as it drags PM inwards through the contracting actomyosin array [14]. We did not observe such coordination in the healing rates of the PM and F-actin wounds (Additional file 12), which again suggests that cells from our wounding assay did not heal according to the classical model of actomyosin arraymediated contraction. It should be noted however, that our assays prioritized the visualization of cytoskeletal events, and as such did not set out to verify the presence of a de facto membrane patch as observable in the Xenopus model or of any of the exocytosis or "explocytosis" events that have been shown to be required for its formation [3]. 




\section{Conclusions}

In mammalian somatic cells, repair of cytoskeletal wounds up to $10 \mu \mathrm{m}$ is not strictly dependent on the assembly and contraction of an actomyosin array.

\section{Limitations}

Apical and basal side of cells cultured in 2D probably exhibit differences in the properties of their cortex cytoskeleton structures or in their local tension. 


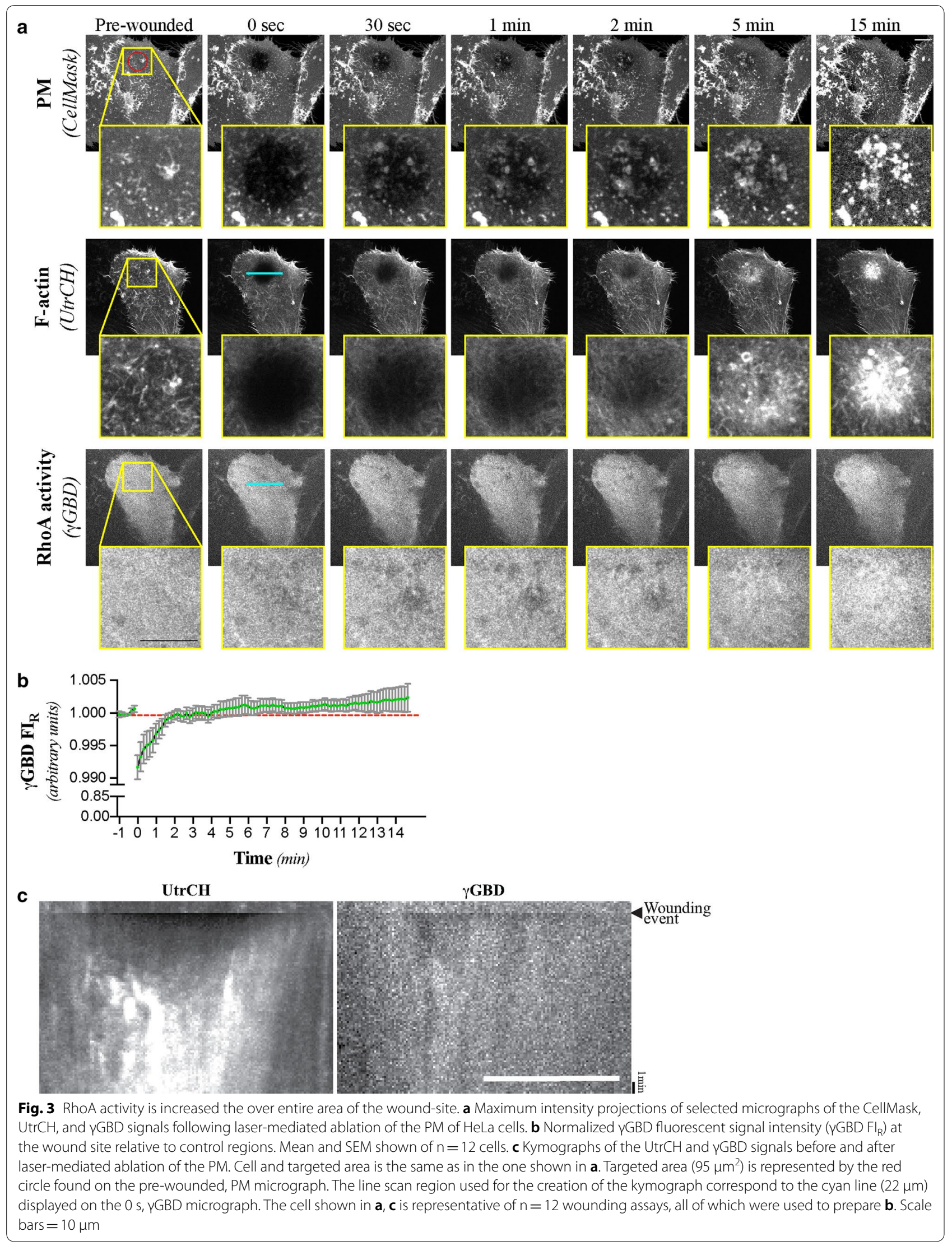


Exclusive targeting of the basal surface may have biased our results and limited compatibility with previously published mechanically wounded-cells or of the repair processes related to the apical surface.

\section{Additional files}

Additional file 1. Rate of change of cytoplasmic FM1-43 fluorescence intensity $(\triangle \mathrm{FM} 1-43 \mathrm{cFI})$ in intact and wounded cells. In intact cells (doted light blue; $\mathrm{n}=3$ ), the rate of FM1-43 FI increase is very low and remains constant. In laser-ablated cells (solid green line; $n=10$ ), the rate of FM1-43 $\mathrm{Fl}$ is significantly increased following ablation, then returns to unwounded rates $20 \mathrm{~s}$ following ablation. Mean and SEM shown.

Additional file 2. Representative line scans of UtrCH Fl at the wound sites of laser-ablated cells. A, C: Micrographs and associated kymographs of the UtrCH signal before and after laser-mediated ablation of the PM. B, D: Linescans associated with the kymographs shown in A and C. Micrographs and kymographs are representative of $n=37$ wounding assays.

Additional file 3. Laser-mediated ablation of the PM does not lead to increased Cdc42 activity. A: Maximum intensity projections of selected micrographs of the CellMask and $\omega G B D$ signals following laser-mediated ablation of the PM of HeLa cells. B: Normalized $\omega G B D$ fluorescent signal intensity $\left(\omega G B D F I_{R}\right)$ at the wound site relative to control regions. Mean and SEM shown of $n=13$ cells.

Additional file 4. Time-lapse video showing actin polymerisation at the site of laser-mediated injury. The video was prepared from maximum intensity projections of micrographs collected from 1 min before wounding, up to $15 \mathrm{~min}$ and $10 \mathrm{~s}$ after the wounding event, at a $10 \mathrm{~s}$ interval (98 micrographs/fluorescence channel) of the cell shown in Fig. 1. Signals corresponding the PM (cell mask) and F-actin $(\mathrm{Utr} \mathrm{CH})$ are shown to the left and right of the composite video, respectively

Additional file 5. Time-lapse video showing increased RhoA activity. The video was prepared from maximum intensity projections of micrographs collected $1 \mathrm{~min}$ before wounding, up to $15 \mathrm{~min}$ and $10 \mathrm{~s}$ after the wounding event, at a 10 s interval (98 micrographs/fluorescence channel) of the cell shown in Fig. 3. Signals corresponding the PM (cell mask), F-actin $(\mathrm{Utr} C \mathrm{H})$, and RhoA activity ( $\gamma \mathrm{GBD})$ are shown to the left, center and right of the composite video, respectively.

Additional file 6. Time-lapse video showing microtubules growing inward from the wound-edge following laser-mediated injury. The video was prepared from maximum intensity projections of micrographs collected $30 \mathrm{~s}$ before wounding, up to $19 \mathrm{~min}$ and $20 \mathrm{~s}$ after the wounding event, at a 10 s interval (120 micrographs/fluorescence channel) of the cell shown in Additional file 11. Signals corresponding the PM (cell mask) and Microtubules (enconsin) are shown to the left and right of the composite video, respectively.

Additional file 7. Time-lapse video showing lack of noticeable Cdc42 activity following laser-mediated injury. The video was prepared from maximum intensity projections of micrographs collected 1 min before wounding, and up to $15 \mathrm{~min}$ and $10 \mathrm{~s}$ after the wounding event, at a 10 s interval (98 micrographs/fluorescence channel) of the cell shown in Additional file 3. Signals corresponding the PM (cell mask), F-actin (UtrCH), and $\mathrm{Cd} c 42$ activity ( $\omega \mathrm{GBD}$ ) are shown to the left, center and right of the composite video, respectively.

Additional file 8. Time-lapse video of PM repair and actin polymerisation shown in Additional file 2A. The video was prepared from maximum intensity projections of micrographs collected from $1 \mathrm{~min}$ before wounding, up to $15 \mathrm{~min}$ and $10 \mathrm{~s}$ after the wounding event, at a $10 \mathrm{~s}$ interval (98 micrographs/fluorescence channel) of the cell shown in Additional file 2A. Signals corresponding the PM (cell mask) and F-actin $(\mathrm{UtrCH})$ are shown to the left and right of the composite video, respectively.
Additional file 9. Time-lapse video of PM repair and actin polymerisation shown in Additional file 2B. The video was prepared from maximum intensity projections of micrographs collected from 1 min before wounding, up to $15 \mathrm{~min}$ and $10 \mathrm{~s}$ after the wounding event, at a $10 \mathrm{~s}$ interval ( 98 micrographs/fluorescence channel) of the cell shown in Additional file 2B. Signals corresponding the PM (cell mask) and F-actin $(\mathrm{UtrCH})$ are shown to the left and right of the composite video, respectively.

Additional file 10. Time-lapse video showing myosin does not accumulate at the wound edges following laser-mediated injury. The video was prepared from maximum intensity projections of micrographs collected from $10 \mathrm{~s}$ before wounding, up to $20 \mathrm{~min}$ and $50 \mathrm{~s}$ after the wounding event, at a 10 s interval (127 micrographs /fluorescence channel) of the cell shown in Fig. 2. Signals corresponding the PM (cell mask) and myosin (MRLC) are shown to the left and right of the composite video, respectively.

Additional file 11. Microtubules grow inward from the wound-edge following laser-mediated ablation of the PM. A: Maximum intensity projections of selected micrographs of the CellMask and Enconsin signals following laser-mediated ablation of the PM of HeLa cells. B: Kymographs of the UtrCH and enconsin signals before and after laser-mediated ablation of the PM. C: Resliced cortical volume (each $10 \mu \mathrm{m}$ ) of selected time-points of the wounding assay shown in A and C. Cell and targeted area is the same as in the one shown in A. Targeted area $\left(95 \mu \mathrm{m}^{2}\right)$ is represented by the red circle found on the pre-wounded, PM micrograph. The line scan region used for the creation of the kymograph correspond to the cyan line $(22 \mu \mathrm{m})$ displayed on the $0 \mathrm{sec}$, F-actin micrograph. The cell shown in $A$ and $C$ is representative of $n=5$. Scale bars $=10 \mu \mathrm{m}$.

Additional file 12. PM and Cytoskeletal response to PM wounding. A: The area of the dark region in the F-actin signal (solid light green line) is significantly bigger than the area of the dark region in the PM signal (dotted red line) for the first $60 \mathrm{~s}$ after wounding. Mean and SEM shown, $\mathrm{n}=14$ cells. $\mathrm{B}$ : The rate of change of the F-actin signal dark area (solid light green line) is significantly different from the rate of change of the PM signal dark area (dotted red line) across the fist 30 s post-ablation. Mean and SEM shown, $n=14$ cells

\section{Abbreviations}

CR: contractile ring; Cdc42: cell division control protein 42 homolog; Drosophila: Drosophila melanogaster; F-actin: filamentous actin; PM: plasma membrane; MT: microtubule; MRLC: myosin regulatory light chain; N-WASP: neural WiskottAldrich syndrome protein; RhoA: Ras homolog family member A; Rho GTPase: Rho family of GTP hydrolases; Xenopus: Xenopus laevis.

\section{Acknowledgements}

We thank David Zhou and Quentin Basiren for their critical revision of the manuscript.

\section{Authors' contributions}

This study was primarily designed by CAM and EB, with input from CD. Data acquisition was performed by CD, assisted by LGB. Analysis was performed by $C D$, assisted by LGB and EB. The manuscript was prepared by $C D$ and EB, with input from LGB and CAM. All authors read and approved the final manuscript.

\section{Funding}

Natural Sciences and Engineering Research Council of Canada (NSERC) Grant Number: 231925.

\section{Availability of data and materials}

Raw images used to produce the data are available from the corresponding author on reasonable request.

Ethics approval and consent to participate

Not applicable.

Consent for publication

Not applicable. 


\section{Competing interests}

The authors declare that they have no competing interests.

Received: 1 June 2019 Accepted: 5 July 2019

Published online: 16 July 2019

\section{References}

1. Riske KA, Dimova R. Electro-deformation and poration of giant vesicles viewed with high temporal resolution. Biophys J. 2005;88(2):1143-55.

2. Hoffman JF. On red blood cells, hemolysis and resealed ghosts. Adv Exp Med Biol. 1992;326:1-15.

3. Davenport NR, Sonnemann KJ, Eliceiri KW, Bement WM. Membrane dynamics during cellular wound repair. Mol Biol Cell. 2016;27(14):2272-85.

4. Togo T, Alderton JM, Steinhardt RA. The mechanism of cell membrane repair. Zygote. 2000;8(Suppl 1):S31-2.

5. Mandato CA, Bement WM. Contraction and polymerization cooperate to assemble and close actomyosin rings around Xenopus oocyte wounds. J Cell Biol. 2001;154(4):785-97.

6. Boucher E, Mandato CA. Plasma membrane and cytoskeleton dynamics during single-cell wound healing. Biochim Biophys Acta. 2015;1853(10 Pt A):2649-61

7. McNeil PL, Vogel SS, Miyake K, Terasaki M. Patching plasma membrane disruptions with cytoplasmic membrane. J Cell Sci. 2000;113(Pt 11):1891-902

8. Chen X, Leow RS, Hu YX, Wan JMF, Yu ACH. Single-site sonoporation disrupts actin cytoskeleton organization. J R Soc Interface. 2014;11(95):20140071.

9. Biro EN, Venyaminov SY. Depolymerization of actin in concentrated solutions of divalent metal chlorides. Acta Biochim Biophys Acad Sci Hung. 1979;14(1-2):31-42.

10. Lemichez E, Gonzalez-Rodriguez D, Bassereau P, Brochard-Wyart F. Transcellular tunnel dynamics: control of cellular dewetting by actomyosin contractility and I-BAR proteins. Biol Cell. 2013;105(3):109-17.

11. Bement WM, Mandato CA, Kirsch MN. Wound-induced assembly and closure of an actomyosin purse string in Xenopus oocytes. Curr Biol. 1999:9(11):579-87.
12. Mandato CA, Bement WM. Actomyosin transports microtubules and microtubules control actomyosin recruitment during Xenopus oocyte wound healing. Curr Biol. 2003;13(13):1096-105.

13. Idone V, Tam C, Goss JW, Toomre D, Pypaert M, Andrews NW. Repair of injured plasma membrane by rapid Ca2+-dependent endocytosis. J Cell Biol. 2008;180(5):905-14.

14. Abreu-Blanco MT, Verboon JM, Parkhurst SM. Cell wound repair in Drosophila occurs through three distinct phases of membrane and cytoskeletal remodeling. J Cell Biol. 2011;193(3):455-64.

15. Bement WM, Sokac AM, Mandato CA. Four-dimensional imaging of cytoskeletal dynamics in Xenopus oocytes and eggs. Differentiation. 2003;71(9-10):518-27.

16. Jaiswal JK, Nylandsted J. S100 and annexin proteins identify cell membrane damage as the Achilles heel of metastatic cancer cells. Cell Cycle. 2015;14(4):502-9.

17. Godin LM, Vergen J, Prakash YS, Pagano RE, Hubmayr RD. Spatiotemporal dynamics of actin remodeling and endomembrane trafficking in alveolar epithelial type I cell wound healing. Am J Physiol Lung Cell Mol Physiol. 2011;300(4):L615-23.

18. Horn A, Van der Meulen JH, Defour A, Hogarth M, Sreetama SC, Reed A, et al. Mitochondrial redox signaling enables repair of injured skeletal muscle cells. Sci Signal. 2017;10(495):eaaj1978.

19. Benink HA, Bement WM. Concentric zones of active RhoA and Cdc42 around single cell wounds. J Cell Biol. 2005;168(3):429-39.

20. Simon CM, Vaughan EM, Bement WM, Edelstein-Keshet L. Pattern formation of Rho GTPases in single cell wound healing. Mol Biol Cell. 2013;24(3):421-32.

21. Togo T. Disruption of the plasma membrane stimulates rearrangement of microtubules and lipid traffic toward the wound site. J Cell Sci. 2006;119(Pt 13):2780-6.

\section{Publisher's Note}

Springer Nature remains neutral with regard to jurisdictional claims in published maps and institutional affiliations.
Ready to submit your research? Choose BMC and benefit from:

- fast, convenient online submission

- thorough peer review by experienced researchers in your field

- rapid publication on acceptance

- support for research data, including large and complex data types

- gold Open Access which fosters wider collaboration and increased citations

- maximum visibility for your research: over $100 \mathrm{M}$ website views per year

At BMC, research is always in progress.

Learn more biomedcentral.com/submissions 